\title{
Identifying student opinion leaders to lead e-cigarette interventions: protocol for a randomized controlled pragmatic trial
}

Kar-Hai Chu ${ }^{1 *}$ D, Sara Matheny ${ }^{1}$, Alexa Furek ${ }^{1}$, Jaime Sidani ${ }^{1}$, Susan Radio ${ }^{2}$, Elizabeth Miller ${ }^{3}$, Thomas Valente ${ }^{4}$ and Linda Robertson ${ }^{2}$

\begin{abstract}
Background: After the US Surgeon General declared youth electronic cigarette (e-cigarette) use an epidemic in 2018, the number of youth e-cigarette users continued to surge, growing from 3.8 million in 2018 to over 5 million 2019. Youth who use e-cigarettes are at a substantially higher risk of transitioning to traditional cigarettes, becoming regular cigarette smokers, and increasing their risk of developing tobacco-related cancer. A majority of youth are misinformed about e-cigarettes, often believing they are not harmful or contain no nicotine. Middle school students using e-cigarettes have been affected by its normalization leading to influence by their peers. However, social and group dynamics can be leveraged for a school-based peer-led intervention to identify and recruit student leaders to be anti-e-cigarette champions to prevent e-cigarette initiation. This study outlines a project to use social network analysis to identify student opinion-leaders in schools and train them to conduct anti-e-cigarette programming to their peers.
\end{abstract}

Methods: In the 2019-2020 academic school year, 6th grade students from nine schools in the Pittsburgh area were recruited. A randomized controlled trial (RCT) was conducted with three arms-expert, elected peer-leader, and random peer-leader-for e-cigarette programming. Sixth grade students in each school completed a network survey that assessed the friendship networks in each class. Students also completed pre-intervention and postintervention surveys about their intention-to-use, knowledge, and attitudes towards e-cigarettes. Within each peerled arm, social network analysis was conducted to identify peer-nominated opinion leaders. An e-cigarette prevention program was administered by (1) an adult content-expert, (2) a peer-nominated opinion leader to assigned students, or (3) a peer-nominated opinion leader to random students.

Discussion: This study is the first to evaluate the feasibility of leveraging social network analysis to identify 6th grade opinion leaders to lead a school-based e-cigarette intervention.

Trial registration: ClinicalTrials.gov NCT04083469. Registered on September 10, 2019.

Keywords: Social network analysis, Tobacco, School, E-cigarette

* Correspondence: chuk@pitt.edu

${ }^{1}$ University of Pittsburgh, Pittsburgh, PA, USA

Full list of author information is available at the end of the article

C C The Author(s). 2021 Open Access This article is licensed under a Creative Commons Attribution 4.0 International License, which permits use, sharing, adaptation, distribution and reproduction in any medium or format, as long as you give appropriate credit to the original author(s) and the source, provide a link to the Creative Commons licence, and indicate if changes were made. The images or other third party material in this article are included in the article's Creative Commons licence, unless indicated otherwise in a credit line to the material. If material is not included in the article's Creative Commons licence and your intended use is not permitted by statutory regulation or exceeds the permitted use, you will need to obtain permission directly from the copyright holder. To view a copy of this licence, visit http://creativecommons.org/licenses/by/4.0/ The Creative Commons Public Domain Dedication waiver (http://creativecommons.org/publicdomain/zero/1.0/) applies to the data made available in this article, unless otherwise stated in a credit line to the data. 


\section{Background}

Electronic cigarette (e-cigarette) use has been rapidly growing among adolescents. The 2019 National Youth Tobacco Survey found current e-cigarette use among middle school students increased from 4.9 to $10.5 \%$ [1]. This increase reversed years of decline in overall youth tobacco product use and occurred despite the US Surgeon General declaring e-cigarette use among youth a national epidemic the year prior [2]. Studies have consistently found that ecigarettes may facilitate the uptake of traditional cigarette smoking among otherwise never-smoking youth [3-7]; additionally, a recent outbreak of severe pulmonary illness has been linked to e-cigarette use.

Nationally representative data regarding perceptions, knowledge, and attitudes surrounding e-cigarettes suggest a simultaneous need and opportunity to conduct school-based education programs on e-cigarettes for youth. Approximately $80 \%$ of youth do not think regular e-cigarette use is harmful [8], and many have limited awareness of nicotine content. The e-cigarette company JUUL, which owns $70 \%$ of the e-cigarette market, advertises enhanced nicotine delivery; yet in a sample of 15-24 year olds who used a JUUL product in the past 30 days, only $37 \%$ knew that its cartridges always contain nicotine [9]. Awareness is also lacking for parents and teachers, increasing the risk of continued misinformation being provided to youth. Focus groups have found that most adults are generally unaware of the ingredients in e-cigarettes [10]. Although $88 \%$ of high school teachers and administrators reported being somewhat or very concerned about e-cigarette use by students, $34 \%$ of schools reported no formal communication from the school to parents about e-cigarette use [11].

Schools play an important role in initiation and prevention of nicotine and tobacco use among adolescents. Previous research suggests that higher rates of school smoking prevalence are associated with an increased risk of adolescent smoking [12, 13]. This influence may occur through a variety of mechanisms, including within school norms regarding tobacco, peer networks, socioeconomic characteristics, misconceptions about usage, and school-specific tobacco control policies [14-16]. However, schools also present an opportunity for intervention, as smoking-prevention programs can be administered in an environment where students are comfortable learning [17]. Simulation models have found that school-based interventions can reduce long-term nicotine dependency in a population [18].

Social network influences can have an effect on adolescent tobacco use. Friends within social networks can be leveraged to produce behavioral changes [19]. This is particularly true for youth, as they tend to emulate behavior of those whom they consider as a friend [20]. Further, network-based interventions can be provided in group settings, which help to reinforce adoption of behavioral change [19].

Using opinion leaders is an effective method of conducting school-based health programs. Intervention programs that are administered by central authority figures are met with more resistance when compared with opinion leaders that are peers [19]. Opinion leaders (also referred to as peer leaders or champions) are able to help modify social norms, which can change perceptions surrounding tobacco products [15]. These changes can be more resilient because the individual experiences changes in the behaviors that they perceive as normal [21].

Social network analysis (SNA) can effectively identify opinion leaders, where various methods can be used to identify, select, and train opinion leaders for health behavior interventions [22]. Identifying opinion leaders through SNA have the benefits of being informed by the full communication and social relational structure of the community as well as implementing a strategy that pairs leaders with those closest to them.

\section{Study objectives}

This primary objective of our study was to assess the feasibility and acceptability of using SNA to identify student opinion leaders in schools and train them to conduct e-cigarette prevention programming for their peers.

\section{Methods}

\section{Trial design}

We conducted an open randomized controlled trial (RCT) with three arms-expert, elected peer-leader, and random peer-leader-for e-cigarette prevention programming. For additional information on this study's compliance with Standard Protocol Items: Recommendations for Interventional Trials (SPIRIT), see Fig. 1 and Supplemental material.

\section{Participants and recruitment}

We recruited nine schools in the Pittsburgh area comprised of 6th grade students (usually 11-12 years old) that are a heterogenous mix of gender, racial, and economic statuses (Table 1). Schools were conveniently selected based on referrals from colleagues that have existing relationships with administrators within each schools. No restrictions were placed on schools for other interventions being conducted. KHC and SM conducted informational meetings that typically included the principal, the guidance counselor, and the teacher of the class in which the intervention would occur. Following the meeting, the principal or classroom teacher would bring the proposed intervention to the school board. Upon approval from the school board, the research team 


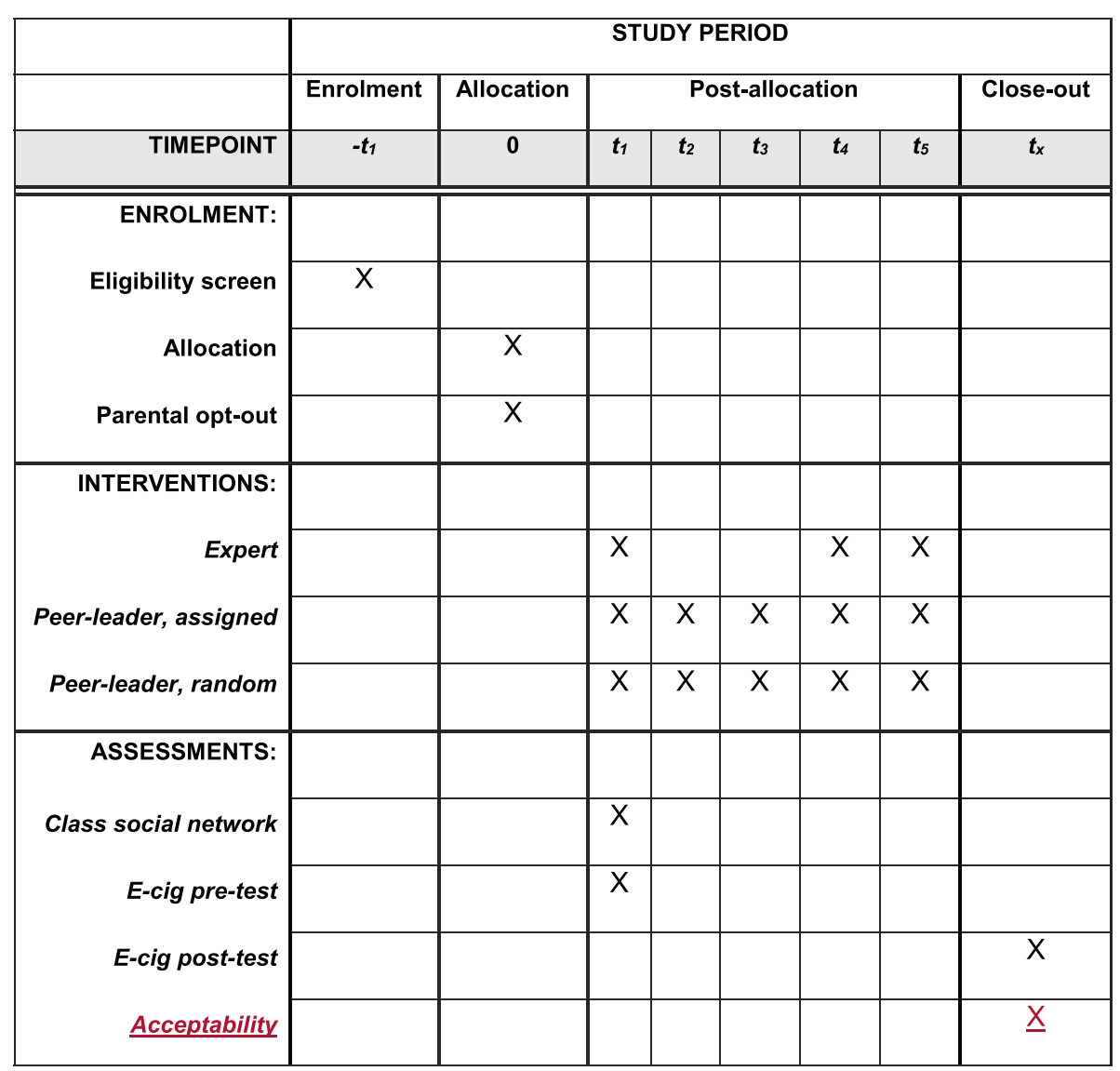

Fig. 1 SPIRIT schedule of enrolment, interventions, and assessments

worked with the schools to schedule days for the intervention at their convenience.

\section{Randomization}

An independent statistician generated an allocation table using Stata's ralloc function, with schools stratified by demographic composition (delineated by greater or less than $67 \%$ white). That allocation table was provided to a research assistant, who assigned schools into the

Table 1 School demographics

\begin{tabular}{llllllllll}
\hline & \multicolumn{1}{l}{ Schools } & & & & & & & \\
\cline { 2 - 10 } & S1 & S2 & S3 & S4 & S5 & S6 & S7 & S8 & S9 \\
\hline Female (\%) & 50 & 47 & 50 & 50 & 50 & 50 & 53 & 50 & 43 \\
White (\%) & 90 & 66.4 & 32 & 63 & 84 & 93 & 93 & 88 & 84.7 \\
Black (\%) & 4 & 22 & 65 & 32 & 6.8 & $<1$ & 2 & 2.8 & 8.5 \\
Hispanic (\%) & $<1$ & 1.3 & 2 & $<1$ & $<1$ & $<1$ & 1 & 2.5 & $<1$ \\
Asian (\%) & 1 & $<1$ & $<1$ & $<1$ & $<1$ & $<1$ & $<1$ & 1.7 & $<1$ \\
Free lunch eligible (\%) & 45 & 57 & 100 & 98 & 50 & 49 & 39 & 29 & 30 \\
6th grade students & 126 & 174 & 266 & 98 & 231 & 93 & 92 & 194 & 299 \\
\hline
\end{tabular}

allocation table based on the order of date of agreement to participate in the study.

\section{Data collection and management}

Consent documents were provided to students in paper form. A letter was sent to all 6th grade students' parents/guardians describing the study. Parents/guardians were given a copy of each survey their child was asked to complete as well as an opt-out form should they choose not to have their child participate. Students provided verbal assent before the study began and were informed they could discontinue without penalty at any time. Students were not individually compensated for participation in the intervention; each participating class was provided with a party at the end of their participation. Additionally, each school that served as a study site was provided an honorarium.

All surveys were conducted by a member of the research team during regular school hours in a standard class period. Surveys were provided to students in paper form. The network survey and baseline e-cigarette survey were administered to students during the first session for all schools regardless of assignment. The final 
e-cigarette surveys were administered at the last day after the program had been completed (Fig. 1). Classroom teachers assisted the study team to ensure completion of surveys.

The principal investigator had the primary responsibility for study monitoring. Quarterly assessments were made of data quality and timeliness, participant risk/ benefit ratio, protection of confidentiality of information, and any other factors that affected the study. The Institutional Review Board (IRB) would be informed immediately on a case by case basis, of any adverse outcomes, while requests for modifications of the protocol would be submitted to the IRB on a quarterly basis.

\section{Sample size}

Given the nature of this study as a feasibility trial, sample size, and power calculations were not considered. The number of students were a convenience sample based on each of the nine schools (Table 1).

\section{Intervention overview}

We based the e-cigarette prevention program on a combination of three existing programs: (1) UPMC Hillman Cancer Center Healthy Choices for Students School Program; (2) CATCH My Breath, an e-cigarette prevention for middle and high school students [23]; (3) Stanford's Tobacco Prevention Toolkit [24]. This pilot study focused on modules that are most effective in reducing youth tobacco use, such as counter-marketing techniques, developing confidence, refusal skills, selfregulation, and normative education [23, 25-27].

Sixth grade students in each school completed a network survey that assessed the social networks in each class. Students listed the Roster ID numbers of up to five other students in four different network-based categories: closest friends, students that they think are the most popular, students that they view as the best leaders, and students they seek advice for personal concerns. Students also completed a pre-test e-cigarette survey. The items on the e-cigarette survey were adapted from the National Youth Tobacco Survey. For each school in peer-leader arm, we analyzed data from the social network survey to identify peer leaders in each classroom (schools in the expert-led arm were still given the social network survey). Once the peer-leaders were identified, we conducted 2 days of training to provide the skills necessary for them to lead other students in e-cigarette prevention programming. After completing the training, peer-leaders were assessed for their knowledge and understanding of the intervention.

During the 2-day intervention program, students in schools assigned to the elected peer-leader arm received the intervention from the trained student-nominated peer-leader (i.e., non-opinion leaders were matched with an opinion leader based on SNA). Students in schools assigned to the random peer-leader arm also received the intervention from the trained student-nominated opinion leader; however, the peer-leaders were randomly assigned. Students in schools assigned to the expert arm were administered the intervention by one of the study team members.

\section{Social network analysis}

Within each class, we used degree centrality as the primary metric to identify peer leaders who are most likely to be influential change agents or promote attitudinal and behavioral change regarding e-cigarette use. Peer leaders were selected based on a weighted in-degree centrality ranking. Both the size of each group and the number of opinion leaders were informed by SNA and varied in each class; however, approximately 4-6 students were targeted to each peer-leader. Each of the nomination categories (i.e., friendship, opinion, academic support, and personal support) were evaluated to ensure the robustness of identification.

\section{Outcomes}

Primary outcomes are satisfaction with the intervention program, including (1) appropriateness of the lessons, (2) peer-leader confidence, (3) students' opinions of the lessons, and (4) student recruitment and retention rates. These data will be collected immediately after completion of the program. Secondary outcomes include intention-to-use and knowledge about e-cigarettesbased on items from the National Youth Tobacco Survey-will be collected before and after the intervention (Fig. 1).

\section{Data analysis}

We will evaluate statistical properties of all data collected, including searching for outliers and missing data. As this study seeks to assess acceptability and feasibility, the primary data will include (1) recruitment rates, (2) retention rates, and (3) satisfaction with the intervention.

\section{Power calculations}

As this study is a feasibility trial, it is inappropriate to consider mean outcomes due to lack of power. However, we will still calculate the standard deviation of the differences between the intervention and control groups.

\section{Discussion}

This pilot trial is the first to evaluate the feasibility of leveraging social network analysis to identify 6th grade opinion leaders to lead a school-based e-cigarette intervention. The project was conducted in nine schools in the Pittsburgh area. We chose a pragmatic approach in 
order to inform implementation-related metrics for future studies to address the e-cigarette epidemic in US youth. If the pilot is successful and signals positive results, a larger fully-powered trial will be pursued to test the effectiveness of a peer-based approach.

The increase of teen e-cigarette use has the ability to create a new generation of nicotine-addicted persons. These nicotine-dependent youth have significantly greater odds of transitioning to traditional cigarettes compared to e-cigarette non-users, increasing their risk of cancer and cancer-related mortalities [19]. Previous research stresses the importance of preventing adolescent tobacco use as a method to prevent cancers and other chronic morbidities later in life. Using opinion leaders is an effective method of conducting schoolbased health programs. Preventing teen e-cigarette through innovative school-based programming can reduce the associated risks of developing tobacco-related cancer [17].

\section{Trial status}

Protocol version: 4

Trial registration: ClinicalTrials.gov

Registration number: NCT04083469 (https://clinicaltrials. gov/ct2/show/NCT04083469).

Date of trial registration: September 10, 2019

Was this trial prospectively registered? Yes

Date recruitment began: August 2019

Completion date: July 2020

\section{Supplementary Information}

The online version contains supplementary material available at https://doi. org/10.1186/s13063-020-04990-z.

\section{Additional file 1.}

\section{Acknowledgements}

We acknowledge Michelle Woods for editorial assistance.

\section{Dissemination policy}

The Principle Investigator (PI), Dr. Chu, registered the study on ClinicalTrials. gov within the first 6 months of study initiation and prior to enrollment of the first subject. In addition, the PI will ensure that study results are posted on ClinicalTrials.gov upon completion of analysis. The University of Pittsburgh Human Research Protection Office has an internal policy in place to ensure that clinical trials registration and results reporting occur in compliance with these policy requirements.

\section{Authors' contributions}

KC initiated the study, developed the study design, and drafted the original manuscript. SM and AF contributed to the data analysis, study implementation, and contributed to the manuscript. SR and JS contributed to the study implementation and contributed to the manuscript. EM, TV, and LR advised on study design and contributed to the manuscript. All authors have agreed to publication.

\section{Funding}

This project was supported in part by award number P30CA047904 and K07CA222338 from the National Cancer Institute. The funders had no role in the study design, data collection and analysis, decision to publish, or preparation of the manuscript. Activities are conducted under the sponsorship of the University of Pittsburgh.

\section{Availability of data and materials}

The datasets used and/or analyzed during the current study are available from the corresponding author on reasonable request.

\section{Ethics approval and consent to participate}

This study was approved by the University of Pittsburgh Institutional Review Board (STUDY19040095). Parents were sent a letter explaining the research and the questionnaires and assessments their child will be asked to complete. Parents were also provided with the principal investigator's contact information and encouraged to call if they had questions or concerns. The letter included a form that parents completed and returned to the PI to opt their child out of participation. Any changes to the protocol or severe adverse events will be reported to the University of Pittsburgh Institutional Review Board.

\section{Consent for publication}

Not applicable.

\section{Competing interests}

There are no conflicts of interest at this time.

\section{Author details}

${ }^{1}$ University of Pittsburgh, Pittsburgh, PA, USA. ${ }^{2}$ UPMC Hillman Cancer Center, Pittsburgh, PA, USA. ${ }^{3}$ UPMC Children's Hospital, Pittsburgh, PA, USA.

${ }^{4}$ University of Southern California, Los Angeles, CA, USA.

Received: 30 May 2020 Accepted: 21 December 2020

Published online: 06 January 2021

\section{References}

1. Cullen KA, Gentzke AS, Sawdey MD, et al. E-cigarette use among youth in the United States, 2019. JAMA. 2019;322:2095. https://doi.org/10.1001/jama. 2019.18387.

2. Stein R. Vaping "epidemic" among youths declared by U.S. Surgeon General. NPR. 2018. Available from: https://www.npr.org/sections/health-shots/201 8/12/18/677755266/surgeon-general-warns-youth-vaping-is-now-an-epidemic.

3. Primack BA, Shensa A, Sidani JE, et al. Initiation of traditional cigarette smoking after electronic cigarette use among tobacco-naïve US young adults. Am J Med. 2018;131:443.e1-9. https://doi.org/10.1016/J.AMJMED.2017.11.005.

4. Primack BA, Soneji S, Stoolmiller M, et al. Progression to traditional cigarette smoking after electronic cigarette use among US adolescents and young adults. JAMA Pediatr. 2015;169:1018-23. https://doi.org/10.1001/ jamapediatrics.2015.1742

5. Barrington-Trimis JL, Urman R, Berhane K, et al. E-cigarettes and future cigarette use. Pediatrics. 2016;138:e20160379. https://doi.org/10.1542/peds. 2016-0379.

6. Soneji S, Barrington-Trimis JL, Wills TA, et al. Association between initial use of e-cigarettes and subsequent cigarette smoking among adolescents and young adults. JAMA Pediatr. 2017;171:788. https://doi.org/10.1001/ jamapediatrics.2017.1488

7. Spindle TR, Hiler MM, Cooke ME, et al. Electronic cigarette use and uptake of cigarette smoking: a longitudinal examination of U.S. college students. Addict Behav. 2017;67:66-72. https://doi.org/10.1016/J.ADDBEH.2016.12.009.

8. Miech RA, Johnston LD, O'Malley PM, et al. Monitoring the future national survey results on drug use, 1975-2017: volume I, secondary school students. Ann Arbor: Institute for Social Research, The University of Michigan; 2018.

9. Willett JG, Bennett $\mathrm{M}$, Hair EC, et al. Recognition, use and perceptions of JUUL among youth and young adults. Tob Control. 2019;28:115-6. https:/ doi.org/10.1136/tobaccocontrol-2018-054273.

10. Coleman BN, Johnson SE, Tessman GK, et al. "It's not smoke. It's not tar. It's not 4000 chemicals. Case closed": exploring attitudes, beliefs, and perceived social norms of e-cigarette use among adult users. Drug Alcohol Depend. 2016:159:80-5. https://doi.org/10.1016/j.drugalcdep.2015.11.028.

11. Truth Initiative (2019) How are schools responding to the youth e-cigarette epidemic? https://truthinitiative.org/news/how-are-schools-responding-juuland-youth-e-cigarette-epidemic. Accessed 19 Feb 2019.

12. Alexander C, Piazza M, Mekos D, Valente T. Peers, schools, and adolescent cigarette smoking. J Adolesc Health. 2001;29:22-30. 
13. Ali MM, Dwyer DS. Estimating peer effects in adolescent smoking behavior: a longitudinal analysis. J Adolesc Health. 2009;45:402-8. https://doi.org/10. 1016/J.JADOHEALTH.2009.02.004.

14. Cavazos-Rehg PA, Krauss MJ, Sowles SJ, et al. Multiple levels of influence that impact youth tobacco use. Tob Regul Sci. 2016;2:106-22. https://doi. org/10.18001/TRS.2.2.2.

15. Lipperman-Kreda S, Grube JW. Students' perception of community disapproval, perceived enforcement of school antismoking policies, personal beliefs, and their cigarette smoking behaviors: results from a structural equation modeling analysis. Nicotine Tob Res. 2009;11:531-9. https://doi. org/10.1093/ntr/ntp033.

16. Sheikh A, Vadera S, Ravey M, et al. A social norms approach to changing school children's perceptions of tobacco usage. Health Educ. 2017;117:5309. https://doi.org/10.1108/HE-01-2017-0006.

17. Taylor J, Taylor A, Lewis S, et al. A qualitative evaluation of a novel intervention using insight into tobacco industry tactics to prevent the uptake of smoking in school-aged children. BMC Public Health. 2016;16:539. https://doi.org/10.1186/s12889-016-3205-8.

18. Chu K-H, Shensa A, Colditz J, et al. Integrating social dynamics into modeling cigarette and E-cigarette use. Health Educ Behav. 2020;47:191-201.

19. Schillinger $D$, Ling PM, Fine $S$, et al. Reducing cancer and cancer disparities: lessons from a youth-generated diabetes prevention campaign. Am J Prev Med. 2017;53:S103-13. https://doi.org/10.1016/J.AMEPRE.2017.05.024

20. Valente TW. Network interventions. Science. 2012;337:49-53. https://doi.org/ 10.1126/science.1217330.

21. Gesell SB, Barkin SL, Valente TW. Social network diagnostics: a tool for monitoring group interventions. Implement Sci. 2013;8:116. https://doi.org/ 10.1186/1748-5908-8-116

22. Rogers E. Diffusion of innovations. 5th ed. New York: Free Press; 2003.

23. Franks A, Kelder S, Dino GA, Horn KA. School-based programs: lessons learned from CATCH, Planet Health, and Not-On-Tobacco. In: Hassan A, editor. School nutrition and activity. Oakville: Apple Academic Press; 2015. p. 147-62.

24. Medicine SS of the tobacco prevention toolkit.

25. CATCH CATCH my breath E-cigarette prevention. https://catchinfo.org/ modules/e-cigarettes/. Accessed 15 Apr 2019.

26. McKenzie TL, Stone EJ, Feldman HA, et al. Effects of the CATCH physical education intervention: teacher type and lesson location. Am J Prev Med. 2001;21:101-9. https://doi.org/10.1016/S0749-3797(01)00335-X.

27. Leon $A C$, Davis $L L$, Kraemer HC. The role and interpretation of pilot studies in clinical research. J Psychiatr Res. 2011;45:626-9. https://doi.org/10.1016/j. jpsychires.2010.10.008.

\section{Publisher's Note}

Springer Nature remains neutral with regard to jurisdictional claims in published maps and institutional affiliations.

Ready to submit your research? Choose BMC and benefit from:

- fast, convenient online submission

- thorough peer review by experienced researchers in your field

- rapid publication on acceptance

- support for research data, including large and complex data types

- gold Open Access which fosters wider collaboration and increased citations

- maximum visibility for your research: over $100 \mathrm{M}$ website views per year

At $\mathrm{BMC}$, research is always in progress.

Learn more biomedcentral.com/submissions 